\title{
$\begin{array}{r}\text { WAGENINGEN } \\ \hline\end{array}$
}

The tuberization signal StSP6A represses flower bud development in potato

Plantenga, F. D. M., Bergonzi, S., Abelenda, J. A., Bachem, C. W. B., Visser, R. G. F., Heuvelink, E., \& Marcelis, L. F. M.

This is a "Post-Print" accepted manuscript, which has been published in "Journal of Experimental Botany"

This version is distributed under a non-commercial no derivatives Creative Commons

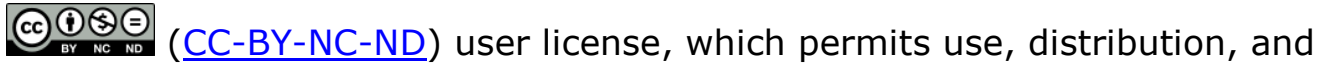
reproduction in any medium, provided the original work is properly cited and not used for commercial purposes. Further, the restriction applies that if you remix, transform, or build upon the material, you may not distribute the modified material.

Please cite this publication as follows:

Plantenga, F. D. M., Bergonzi, S., Abelenda, J. A., Bachem, C. W. B., Visser, R. G. F., Heuvelink, E., \& Marcelis, L. F. M. (2018). The tuberization signal StSP6A represses flower bud development in potato. Journal of Experimental Botany, 420. DOI: $10.1093 /$ jxb/ery420

You can download the published version at:

https://doi.org/10.1093/jxb/ery420 


\section{The tuberization signal StSP6A represses flower bud development in potato}

2

3 Faline D.M. Plantenga ${ }^{1}$, Sara Bergonzi ${ }^{2}$, José A. Abelenda ${ }^{2}$, Christian W.B. Bachem²,

4 Richard G.F. Visser ${ }^{2}$, Ep Heuvelink ${ }^{1}$ and Leo F.M. Marcelis ${ }^{1}$

5

$6 \quad{ }^{1}$ Horticulture and Product Physiology, Wageningen University \& Research, Wageningen, the 7 Netherlands.

$8 \quad$ 2Plant Breeding, Wageningen University \& Research, Wageningen, the Netherlands.

9

10

faline.plantenga@wur.nl - 0031641620926

11 sara.bergonzi@wur.nl

12 jose.abelendavila@wur.nl

13 christian.bachem@wur.nl

14 richard.visser@wur.nl

15 ep.heuvelink@wur.nl

16 leo.marcelis@wur.nl

17

Date of resubmission: 3 November 2018

Number of tables in the main file: 2

Number of figures in the main file: 8 (all figures in color online)

Word count: 6497

Number of tables in the supplementary data: 2

Number of figures in the supplementary data: 2 (all figures in color online) 
TITLE

The tuberization signal StSP6A represses flower bud development in potato

\section{Running title: Tuberization signal represses potato flower development}

\section{HIGHLIGHT}

For the first time it is shown that the tuberization signal StSP6A not only induces tuberization, but also represses flower bud development in potato

\section{ABSTRACT}

Potato (Solanum tuberosum L.) can reproduce sexually through flowering and asexually through tuberization. While tuberization has been thoroughly studied, little research has been done on potato flowering. Flower bud development in the strictly short-day tuberizing $S$. tuberosum group Andigena is impaired under short-day conditions. This impaired development may indicate that tuberization negatively influences flowering. The aim of this research was to determine how tuberization affects flower bud development. To find out whether the absence of tubers improves flowering we prevented tuberization by: (1) grafting potato scions onto wild potato rootstocks, which were unable to form tubers; (2) removing stolons, the underground structures on which tubers form; (3) using plants that were silenced in the tuberization signal StSP6A. Additionally, transgenic plants with increased StSP6A expression were used to determine if flower bud development was impaired. The absence of a tuber-sink alone did not accelerate flower bud development, nor did it allow more plants to reach anthesis (open flowering stage) or have more open flowers. Interestingly, reducing StSP6A expression improved flower bud development, and increasing expression impaired it. Our results show that flower bud development in potato is repressed by the tuberization signal StSP6A, and not by competition with the underground tuber-sink.

Keywords: day length, grafting, potato flowering, stolons, StSP6A, tuberization 
Potato (Solanum tuberosum L.) is the third largest crop for human consumption worldwide and due to its high nutritional value and low production costs, consumption is most certainly expected to increase (International Potato Center, 2016; Zaheer and Akhtar, 2016). Potato plants are able to reproduce both sexually, through flowers, and asexually through the formation of tubers. Although both reproduction methods are present in the plant, most research has been done on tuberization. Commercial potato production mainly uses "seed tubers" and not "true seeds" to propagate plants. Asexual reproduction is used for propagation because potato plants are tetraploid and highly heterozygous. Incorporation of a Sli gene allows for selffertilization of diploid potato lines, which makes the generation of homozygous lines possible (Lindhout et al., 2011). These developments have made hybrid breeding in potato possible and thereby also the use of true potato seeds as starting material. Hybrid breeding of potato will enable breeders to specifically select for desired traits in new varieties and develop these varieties much faster than in traditional potato breeding (Lindhout et al., 2011). The developments in potato breeding and propagation require the understanding of not only tuberization, but also potato flowering.

Whether a potato plant starts to tuberize or flower, depends strongly on environmental cues (Ewing and Struik, 1992; Almekinders and Struik, 1996). Potato tuberization is strongly influenced by day length and is induced under short-day conditions (Batutis and Ewing, 1982). Modern varieties are no longer dependent on short days to tuberize, as breeders have selected against this trait. Nevertheless, the photoperiodic mechanism controlling tuberization remains conserved in all potato plants (Kloosterman et al., 2013). As potato tuberization has been intensively studied, we have a good understanding of the molecular regulation behind this process (Abelenda et al. 2011; Navarro et al., 2011; González-Schain et al. 2012; Navarro et $a l ., 2015)$. The photoperiodic regulation of tuberization strongly resembles the photoperiodic control of flowering time in the model plant Arabidopsis thaliana and other plants (Tsuji et al., 2011; Andrés and Coupland, 2012; Fu et al., 2014). SELF-PRUNING 6A (StSP6A) was identified as a potato homologue of the flowering signal FLOWERING LOCUS T (FT) in A. thaliana and instead of inducing the flower transition, StSP6A induces tuber formation in potato (Potato Genome Sequencing Consortium, 2011; Navarro et al., 2011). After StSP6A is expressed in the leaves, the mobile StSP6A protein moves through the plant to underground stems, called stolons, where it induces tuberization. The cascade of events leading to short-day 
dependent expression of StSP6A mRNA has also been revealed. This control includes the genes CYCLING DOF FACTOR (StCDF1) and CONSTANS (StCO) (Kloosterman et al., 2013), which are also involved in photoperiodic control of flowering in A. thaliana. In potato StCDF1 downregulates StCO, which in turn induces SELF-PRUNING 5G (StSP5G), a repressor of StSP6A (Kloosterman et al., 2013; Abelenda et al., 2016). Within the Solanaceae, the FT family has undergone a large expansion and another homologue of FT called SELF-PRUNING $3 D(S t S P 3 D)$ was found in potato and was proposed to control the flower transition (Potato Genome Sequencing Consortium, 2011; Navarro et al., 2011). However, how this regulation takes place remains to be elucidated.

Although some research has been performed on potato flowering, ambiguity remains concerning the environmental effect on flower transition and whether this is a long-day, shortday or day-neutral processes (Jones and Borthwick, 1938; Almekinders and Struik, 1994; Navarro et al., 2011; González-Schain et al., 2012). Although little is known about the flower transition, it has been established that potato flower development is negatively affected in tuber inducing conditions like short days (Turner and Ewing, 1988; Rodríguez-Falcón et al., 2006; Plantenga et al., 2016). Flower buds abort more frequently and less open flowers are formed. Failure of flower bud development in short days could be due to a direct photoperiod effect, but alternatively might be the result of a negative effect exerted by tuberization. Tubers are strong assimilate sinks (Sweetlove et al., 1998) and may leave insufficient assimilates to support flowering (Almekinders and Struik, 1996). However, previous studies do not agree whether or not flowering competes with tuberization (Krantz, 1939; Thijn, 1954; Jessup, 1958; Krauss and Marschner, 1984; Pallais, 1987).

Here we confirm that while the flower transition occurs independently of photoperiods, later stages of flower bud development are impaired under short-day conditions which induce tuberization. Specifically, we investigated whether flower bud development is impeded by competition for assimilates between flowering and tuberization or by the tuberization signal StSP6A. We performed experiments where we prevented tuberization in three different ways; (1) by grafting potato scions onto wild potato rootstocks, that were unable to form tubers; (2) by removing stolons, the structures on which tubers form; (3) by using transgenic plants that were silenced in the tuberization signal StSP6A (Fig. 1). Finally we demonstrated how increased StSP6A expression affected flower bud development in long days. Together, our experiments show that the tuberization signal StSP6A inhibits flower bud development and only the repression of this signal improves flower bud development. 
MATERIALS AND METHODS

\section{Plant materials}

Solanum tuberosum group Andigena (S. andigena), Solanum tuberosum CE3027 and Solanum tuberosum CE3130 were used. $S$. andigena is a tetraploid, obligatory short-day plant for tuberization. CE3027 and CE3130 are progeny plants from a mapping population that segregates for timing of tuberization (Kloosterman et al., 2013), where CE3027 tuberizes early in short days and late in long days, and CE3130 tuberizes early under both short and long days. These lines were used because they can produce open flowers in our climate chamber conditions, as opposed to $S$. andigena. All genotypes were propagated in vitro and maintained in tissue culture in MS20 medium (Murashige and Skoog, 1962). Additionally, two wild Solanum species that are unable to tuberize were used: Solanum etuberosum (CGN17714) and Solanum palustre (CGN18241) (CGN seedbank, Wageningen, Netherlands). Seeds of these species were disinfected in $2.7 \% \mathrm{NaOCl}$ for 30 minutes, soaked in $700 \mathrm{ppm}$ gibberellic acid $\left(\mathrm{GA}_{3}\right)$ for 24 hours in the dark and sown on MS20. Finally, two StSP6A silenced lines in a $S$. andigena background (StSP6A RNAi \#1 and StSP6A RNAi \#13) and two StCDF1 overexpressing lines in a CE3027 background (35S::StCDF1\#3 and 35S::StCDF1 \#4) were used.

\section{Plant transformation}

In order to generate these lines, StSP6A coding regions were PCR amplified from Solanum tuberosum group Andigena cDNA through Phusion High-Fidelity DNA Polymerase (Thermo Scientific $^{\mathrm{TM}}$ ) using specific primers (RNAi6Afor 5'-CACCTACAAATACAAGCTTTGGAA3' and RNAi6Arev 5'-CTCTATTTATTTATAACAT-3'). Then, cloned in pENTR TM/DTOPO ${ }^{\circledR}$ (Invitrogen) following manufacturer recommendations. The final StSP6A RNAi construct was generated using the StSP $6 A$ pENTR ${ }^{\mathrm{TM}} / \mathrm{D}-\mathrm{TOPO}$ entry clone and further insertion by recombination with the LR clonase ${ }^{\mathrm{TM}}$ II enzyme (Invitrogen) into the pK7GWIWG2(II) vector (Karimi et al., 2002). Transgenic plants bearing the StSP6A RNAi construct were generated by Agrobacterium-mediated transformation of in vitro internodes as described previously in Visser (1991).

The StCDF1.1 coding region was also amplified with Phusion High-Fidelity DNA Polymerase RNAi) and cloned in pENTR ${ }^{\mathrm{TM}} / \mathrm{D}-\mathrm{TOPO}{ }^{\circledR}$ (Invitrogen) as previously described (Kloosterman 
et al., 2013). Binary plasmids were obtained after LR clonase ${ }^{\mathrm{TM}}$ II enzyme (Invitrogen) reaction of StCDF1.1-pENTR ${ }^{\mathrm{TM}} / \mathrm{D}-\mathrm{TOPO}{ }^{\circledR}$ with the $\mathrm{pK} 7 \mathrm{WG} 2$ plasmid, obtaining the 35S::StCDF1.1 plasmid (Karimi et al., 2002). In order to generate 35S::StCDF1 transgenic plants, Agrobacterium-mediated transformation of CE3027 internodes with both plasmids was performed as described in Visser (1991).

S. andigena StSP6A RNAi and CE3027 35S::StCDF1 plantlets were propagated in vitro and grown with the other potato plants.

\section{Growing conditions and measurements}

Exp.1. Removing the tuber-sink: grafting onto a non-tuberizing rootstock

Two grafting experiments were performed in a greenhouse in short- and in long-day conditions. In short days CE3027 scions were grafted onto S. etuberosum and S. palustre rootstocks and vice versa. Also control grafts were made where scions were grafted onto rootstocks of their own genotype. In long days, nine grafting combinations were made between CE3027, CE3130 and S. etuberosum. CE3027 and CE3130 scions were grafted onto $S$. etuberosum rootstocks, $S$. etuberosum scions were grafted onto CE3027 and CE3130 rootstocks, and control grafts were made with scions and rootstocks from the same genotype. Additionally, S. etuberosum scions were grafted onto CE3027 and CE3130 rootstocks, which maintained their leaves, to ensure the production of the tuberization signal StSP6A (climate details in Supplementary Table S1). In vitro plantlets were transplanted to $5 \mathrm{~L}$ pots with a clay-peat mixture. Grafting was done with two-week old CE3027 and CE3130 plants and three-week old S. etuberosum and $S$. palustre plants. The stem was cut after the fourth leaf from the bottom. A splice-graft was made and the rootstock and scion were kept together with silicone grafting clips (Beekenkamp 1.5 $\mathrm{mm}$ and Simonetti $2.9 \mathrm{~mm}$ ). Leaves were removed from the rootstock, unless indicated otherwise. Grafts were placed in a high humidity compartment until the grafting unions had set. The plants were manually watered and fertilized $\left(2 \mathrm{~g} \cdot \mathrm{L}^{-1}\right.$, Osmocote Exact Standard $3-4 \mathrm{M}$, Everris). Flowering and tuberization was determined once a week. Anthesis (opening flowering stage) of the primary stem and the maximum number of open flowers per plant were noted. Tuberization time was determined by carefully checking the stolon tip for swelling. Nine weeks after grafting, the tubers were harvested, oven-dried at $105^{\circ} \mathrm{C}$ and weighed.

\section{Exp. 2. Removing the tuber-sink: removing stolons}

CE3027 plantlets were transplanted to $17 \mathrm{~cm} \varnothing$ pots with a clay-peat mixture and placed in a climate chamber (details in Supplementary Table S1). Plants were grown in short days (8 hours light) under 200 or $400 \mu \mathrm{mol} \cdot \mathrm{m}^{-2} \cdot \mathrm{s}^{-1}$ (photosynthetic photon flux density) light (SD200 and 
SD400 respectively) and in long days (16 hours light) under $200 \mu \mathrm{mol} \cdot \mathrm{m}^{-2} \cdot \mathrm{s}^{-1}$ light (LD200). The high-light short day and low-light long day received the same daily light sum. In half of the plants in each light treatment, stolons were removed as soon as formed, resulting in six treatments in total. Light emitting diodes (LEDs) were used for the lighting (Philips GreenPower LED production module $120 \mathrm{~cm}$ DeepRed/White-2012). Light intensities were measured at the top of the plant canopy with a quantum sensor (LI-COR Biosciences, LI-190SB Quantum, LI-1400 data logger) and corrected by adjusting LED height every two weeks. Plants were rotated three times a week to ensure a homogenous light distribution. Side-shoots were removed. Water was given manually and liquid fertilizer was supplied once per week (EC 2.1 $\mathrm{dS} \mathrm{m}^{-1}, \mathrm{pH} 5.5 ; 1.2 \mathrm{mM} \mathrm{NH}_{4}^{+}, 7.2 \mathrm{mM} \mathrm{K}^{+}, 4.0 \mathrm{mM} \mathrm{Ca}^{2+}, 1.82 \mathrm{mM} \mathrm{Mg}^{2+}, 12.4 \mathrm{mM} \mathrm{NO}_{3}^{-}, 3.32$ $\mathrm{mM} \mathrm{SO}_{4}{ }^{2-}, 10 \mathrm{mM}$ P, $35 \mu \mathrm{M} \mathrm{Fe}^{3+}, 8.0 \mu \mathrm{M} \mathrm{Mn}^{2+}, 5.0 \mu \mathrm{M} \mathrm{Zn}^{2+}, 20 \mu \mathrm{M} \mathrm{B}, 0.5 \mu \mathrm{M} \mathrm{Cu}^{2+}, 0.5 \mu \mathrm{M}$ $\left.\mathrm{MoO}_{4}{ }^{2-}\right)$. Plants were examined three times a week for stolons, flower bud appearance, anthesis, number of flowers and tuberization. A destructive harvest including fresh and dry weight measurements of tubers and shoot (aboveground stem, leaves and shoot apex) was done after eight weeks.

\section{Exp. 3. Removing the tuberization signal: reducing StSP6A expression}

Plants of S. andigena wild-type and two StSP6A RNAi lines (\#1 and \#13) were transplanted to $17 \mathrm{~cm} \varnothing$ pots and placed in a climate chamber (details in Supplementary Table S1). In addition to the three light treatments used in Exp. 2, a long-day treatment of $400 \mu \mathrm{mol} \cdot \mathrm{m}^{-2} \cdot \mathrm{s}^{-1}$ (LD400) was applied. Plants were grown and examined as in Exp. 2. Additionally, flower bud development was recorded (flower bud size was categorized from zero to five where zero was no flower bud and five was an open flower). This was done due to the bad flowering success of $S$. andigena and the low chances of reaching anthesis. A destructive harvest was performed after eight weeks of growing and included fresh and dry weight measurements of tubers and shoot.

Exp. 4. Removing the tuberization signal and tuber sink: reducing StSP6A expression and removing stolons

S. andigena wild-type and StSP6A RNAi \#13 plants were transplanted to $17 \mathrm{~cm} \varnothing$ pots and placed in a short-day chamber with $400 \mu \mathrm{mol} \cdot \mathrm{m}^{-2} \cdot \mathrm{s}^{-1}$ light from fluorescent tubes (Philips; Master TL-D Reflex 58W/840 Coolwhite) (climate details in Supplementary Table S1). In half of the wild-type $S$. andigena plants, stolons were removed. Stolons were also removed in half of the StSP6A RNAi \#13 plants to determine whether stolon removal affected plant growth in non-tuberizing plants. Plant growth control and determination of tuberization time and flower bud appearance were performed as in Exp. 2 and 3. Because flower bud size was only 
categorized and not measured precisely in Exp. 3, flower bud development in Exp. 4 was determined by measuring the diameter of the biggest flower bud on each plant, three times a week.

Exp. 5. Increasing the tuberization signal: overexpressing StCDF1 in long days

An additional experiment was performed to confirm that StSP6A affected flower bud development. Instead of reducing StSP6A in short days, StCDF1 overexpressing lines were used with upregulated StSP6A expression in long days. Eight wild-type CE3027, eight $35 S:: S t C D F 1 \# 3$ and eight $35 S:: S t C D F 1$ \#4 plantlets were transferred to $15 \mathrm{~cm} \varnothing$ pots and placed in a long-day chamber with $200 \mu \mathrm{mol} \cdot \mathrm{m}^{-2} \cdot \mathrm{s}^{-1}$ light from fluorescent tubes (Philips; Master TL-D Reflex 58W/840 Coolwhite) (details climate in Supplementary Table S1). Plant growth control was performed as in Exp. 2, 3 and 4. Photographs of the shoot apex were taken after eight weeks of growing and anthesis was documented.

\section{RNA analysis}

StSP6A expression was analyzed to determine if the StSP6A silenced lines were indeed silenced in StSP6A and if the StCDF1 overexpressing lines had upregulated StSP6A. Furthermore, $S t S P 3 D$ expression was analyzed to determine if the StSP6A silenced lines did not increase expression of the flowering signal StSP3D. Leaf samples of the plants in Exp. 3 were collected after five weeks, just before the first tuberization started. The fifth leaf from the top was sampled one hour after the lights went on. Leaves from three plants were collected, pooled into one sample and frozen in liquid nitrogen and stored at $-80^{\circ} \mathrm{C}$. Leaves were also collected from Exp. 5. The fourth and fifth leaf from the top were collected after five weeks, two hours after lights went on. Two plants were pooled and four pools per genotype were made. Gene expression was determined using qPCR (quantitative reverse transcription polymerase chain reaction). Frozen leaf material was ground and used for RNA extraction with an RNeasy plant mini-kit (Qiagen). A spectrophotometer (NanoDrop, Thermoscientific, Thermofisher) determined RNA concentration and quality. A DNase treatment was performed using Amplification grade DNase I (Invitrogen, Thermofisher). $1 \mu \mathrm{g}$ of RNA was used for cDNA synthesis with an iScript kit (Bio-rad). RNA extraction, DNase treatment and cDNA synthesis were performed as described in the supplied manufacturer's protocols. $20 \mu 1$ of cDNA was diluted to a total volume of $150 \mu 1.5 \mu 1$ of SYBR-green (iQ-SYBR-green super mix, Bio-Rad), $0.25 \mu 1$ Forward Primer $(10 \mu \mathrm{M}), 0.25 \mu 1$ Reverse Primer $(10 \mu \mathrm{M}), 0.5 \mu 1$ Milli-Q water and $4 \mu 1$ diluted cDNA were used for the qPCR. In Exp. 3 three technical replicates were used per pooled sample. Samples were placed in a Thermal Cycler $(\mathrm{C} 1000$, Bio-Rad $)$ set to $95^{\circ} \mathrm{C}$ for 3 minutes, 
40 cycles of $95^{\circ} \mathrm{C}$ for 15 seconds and $60^{\circ} \mathrm{C}$ for 1 minute, followed by $95^{\circ} \mathrm{C}$ for 10 seconds and for a melt curve $65^{\circ} \mathrm{C}$ to $95^{\circ} \mathrm{C}$ in $0.5^{\circ} \mathrm{C}$ steps every 5 seconds. Primers used were: StSP6A (PGSC0003DMT400060057):

GACGATCTTCGCAACTTTTACA, CCTCAAGTTAGGGTCGCTTG and StSP3D (scaffold PGSC0003DMB00000014, unannotated): (F) GGACCCAGATGCTCCAAGTC, (R) CTTGCCAAAACTTGAACCTG and StNAC (reference gene NASCENT POLYPEPTIDE-ASSOCIATED COMPLEX ALPHA, PGSC0003DMT400072220):

ATATAGAGCTGGTGATGACT, TCCATGATAGCAGAGACTA. Primers for StSP6A and StSP3D were used in (Navarro et al., 2011) and the StNAC primer had an efficiency of $99 \%$.

\section{Data analysis}

A student's t-test was used to compare two treatments and a one-way analysis of variance (ANOVA) was used to compare more than two treatments. A Bonferonni pair-wise comparison was used to determine which treatments significantly differed $(\alpha=0.05$, IBM, SPSS Statistics 22 and GenStat, $18^{\text {th }}$ Edition). When data was ordinal or not normally distributed (tested with a Shapiro-Wilk W-test for non-normality in GenStat), a non-parametric Kruskal-Wallis test and Dunn's pairwise comparisons $(\alpha=0.05)$ were computed in SPSS. Comparisons between light treatments in Exp. 2 and 3 were based on biological replicates as independent experimental units. For gene expression analysis three technical replicates were used for the qPCR analysis in Exp. 4 and four biological replicates were used for qPCR analysis in Exp. 5. $100 / 2^{-\Delta \mathrm{Ct}}$ was used to determine gene expression values. $\mathrm{Ct}$ (cycle threshold) values of the gene of interest (StSP6A and StSP3D) were used to determine expression of the gene of interest compared to the housekeeping gene StNAC. Invariant expression of StNAC under the tested conditions is shown in Supplementary Fig. S1.

\section{RESULTS}

\section{Removing the tuber-sink: grafting onto a non-tuberizing rootstock}

In order to establish how the absence or presence of tubers would affect flowering of the scions, two grafting experiments were performed in short-day and long-day conditions.

\section{Grafting under short day conditions}


Short-day conditions strongly promote tuberization. To determine whether flower bud development in CE3027 would improve without tubers, we grafted CE3027 scions onto nontuberizing $S$. etuberosum and $S$. palustre rootstocks and grew them in short-day conditions. CE3027 scions underwent floral transition and as expected the flower buds failed to develop in the control grafts with tuberizing CE3027 rootstocks. Moreover, the buds also failed to develop when the CE3027 scion was grafted onto the non-tuberizing $S$. etuberosum or $S$. palustre rootstocks. Thus, the absence of tubers could not improve flower bud development. Opposite grafts were made with S. etuberosum and S. palustre scions on CE3027 rootstocks to determine how tubers would affect flower bud development. However, in the short-day conditions, neither $S$. etuberosum nor S. palustre transitioned to flowering and the CE3027 rootstocks failed to tuberize. To gain a better understanding on the effect of tubers on flower bud development, and attempt to induce flowering in S. etuberosum, a grafting experiment was performed under long-day conditions.

\section{Grafting under long day conditions}

Grafting CE3027 scions onto non-tuberizing S. etuberosum rootstocks did not improve flower bud development compared to the control grafts, which tuberized (CE3027 scion on CE3027 rootstock) (Fig. 2). Surprisingly, the opposite effect was observed. Although all tested graft combinations underwent flower transition (data not shown), grafts with CE3027 scions on $S$. etuberosum rootstocks only reached anthesis in four of the eight (50\%) plants, whereas nine of the eleven (>80\%) control grafts reached anthesis (Fig. 3). Furthermore, the grafts with $S$. etuberosum rootstocks had almost half the number of open flowers compared to control grafts. The grafts made with scions of the early tuberizing genotype CE3130 also had impaired flower bud development in grafts with non-tuberizing rootstocks (Supplementary Table S2A). To determine if the presence of tubers would impair S. etuberosum flower bud development, we made opposite grafts with S. etuberosum scions on tuberizing CE3027 rootstocks. In contrast to the short-day grafting experiment, flower transition occurred in S. etuberosum and the flower buds developed into open flowers. Furthermore, CE3027 rootstocks tuberized, even when S. etuberosum scions were grafted onto them. However, a larger fraction of grafts with S. etuberosum scions on tuberizing CE3027 rootstocks reached anthesis, than of control grafts with S. etuberosum scions on non-tuberizing S. etuberosum rootstocks (Table 1). When comparing grafts in which the CE3027 rootstock was completely defoliated, with grafts in which some leaves were kept below the graft junction, the presence of leaves accelerated tuberization in CE3027 rootstocks with approximately nine days (data not shown). Also, a 
larger fraction of grafts with leafy CE3027 rootstocks reached anthesis than grafts with leafless CE3027 rootstocks. Grafts with S. etuberosum scions on the early tuberizing CE3130 rootstocks showed a similar result. Anthesis was higher in grafts with CE3130 rootstocks and the presence of leaves accelerated tuberization, and also increased the number of plants with open flowers. (SupplementaryTable S2B). Thus, tuberizing rootstocks did not impair the flower bud development of $S$. etuberosum scions.

Taken together, the interspecific grafting experiments did not show that the presence of tuber sinks impaired flower bud development, but rather had an unexpected opposite outcome where an improved flower bud development was observed in grafts producing tubers. To validate that these results were not due to interspecific interaction in the grafts, we performed another experiment where the tuber-sink was removed within the same genotype.

\section{Removing the tuber-sink: removing stolons}

To determine whether tubers negatively influenced flower bud development, tuberization was prevented by removing the stolons in CE3027 plants. Removing stolons did not significantly affect the number of flowering plants nor the number of open flowers per plant (Table 2). Also, the time until anthesis was not affected by removing the stolons (data not shown). The light conditions under which plants were grown did affect flower bud development. The number of flowering plants was low in low-light short days $\left(200 \mu \mathrm{mol} \cdot \mathrm{m}^{-2} \cdot \mathrm{s}^{-1}\right)$, but in high-light short days $\left(400 \mu \mathrm{mol} \cdot \mathrm{m}^{-2} \cdot \mathrm{s}^{-1}\right)$ the number of flowering plants was almost as high as in long days (200 $\left.\mu \mathrm{mol} \cdot \mathrm{m}^{-2} \cdot \mathrm{s}^{-1}\right)$. Nevertheless, removing stolons did not improve flower bud development, both under short-day and long-day conditions.

These results confirm that short-day conditions impair flower bud development in CE3027. Although the number of flowering plants was similar in a high-light short day compared to a low-light long day (SD400 and LD200 had the same daily light sum), the maximum number of open flowers per plant was significantly higher in the long day treatment (1.2 flowers in SD400 vs. 6.5 in LD200).

Tuberization took place in all light treatments unless stolons were removed (Table 2). The short-day treatment with high light intensity resulted in the fastest tuberization and the highest tuber biomass. Plants without stolons had a higher shoot biomass than plants with stolons. The light treatments with the highest light sum (SD400 and LD200) had a higher shoot biomass than the low light sum short day (SD200), in both tuberizing and non-tuberizing plants. The total biomass (tuber + shoot) was highest in the high-light short-day treatment. 
In summary, preventing tuberization by removing the stolons did not improve flower bud development, even though flower bud development was impaired in short days.

\section{Removing the tuberization signal: reducing $S t S P 6 A$ expression}

To determine whether the tuberization signal negatively influenced flower bud development, we used transgenic plants with reduced expression of the tuberization signal StSP6A. Flower buds were formed in all $S$. andigena plants, but flower bud development of the $S$. andigena wild type was impaired in short days compared to long days (Fig. 4). Wild-type plants under high-light short-day and low-light long-day conditions (SD400 and LD200) received the same daily light sum, but flower buds were smaller in the short-day treatment (Fig. 5A, $P=0.02$ ). In the StSP6A RNAi lines grown under short-day conditions, flower bud development was improved compared to the wild type. Under these conditions, flower bud development in the StSP6A RNAi lines equaled the level of flower bud development in the wild-type plants under long-day conditions (Fig 5A, no significant difference between short day StSP6A RNAi lines and long day wild-type lines, $P=0.12$ ). Moreover, two of the five StSP6A RNAi \#13 plants in the high-light short days reached anthesis, which did not occur in $S$. andigena wild-type or StSP6A RNAi plants in any other treatment, not even in long days (Fig. 5B). In long days, a lower StSP6A expression did not have an effect on flower bud development. Reducing StSP6A expression did not affect the flower transition time in either short or long days. Gene expression analysis of StSP6A in StSP6A RNAi lines show that these lines were indeed silenced in StSP6A (Supplementary Fig. S2A). As expected, tuberization in the transgenic lines with reduced StSP6A expression, was inhibited compared to the wild-type plants (Supplementary Fig. S2B). Wild-type plants in high-light long-day conditions showed a later and reduced tuberization compared to the plants in short days, while low-light long-day plants did not tuberize at all. In summary, our results show that inhibiting tuberization by reducing StSP6A expression in potato plants grown under short-day conditions improves flower bud development.

\section{Removing the tuberization signal and the tuber-sink: reducing StSP6A expression and removing stolons}

As the experiments testing the removal of the tuber-sink used different genotypes than the experiments testing removal of the tuberization signal $S t S P 6 A$, we performed a short-day experiment with $S$. andigena, where the tuberization signal StSP6A and the stolons were removed. Also, flower bud development was measured in more detail, to better illustrate differences between treatments. Plants with reduced levels of StSP6A, clearly developed larger 
407

408

409

410

411

412

413

414

415

416

417

418

419

420

421

422

423

424

425

426

427

428

429

430

431

432

433

434

435

436

437

438

439

440

flower buds than wild-type plants, as in Exp. 3 (Fig. 6). The only plant to reach anthesis was a StSP6A RNAi plant without stolons. However, only removing the stolons did not significantly affect the flower bud size. Reducing StSP6A expression or removing the stolons did not affect the flower bud appearance time, which occurred on average after 28 days in all treatments (data not shown). The results show flower bud development is improved when the tuberization signal is removed, but not when only tubers are removed.

\section{Increasing the tuberization signal: overexpressing $S t C D F 1$ in long days}

StCDF1 overexpressing lines in a CE3027 background were used to confirm that StSP6A impairs flower bud development. Both StCDF1 overexpressing lines in long days had upregulated StSP6A expression compared to the wild type (Fig. 7A). The flower bud development in these lines was inhibited and no plants reached anthesis (zero of the 16 plants) (Fig. 7B). The wild-type CE3027 plants were able to reach anthesis in long days (five of the seven plants had open flowers, one plant died).

\section{All experiments: plant growth after removing the tuber-sink}

In the experiments where tubers were removed, but the plants remained induced to tuberize, the plants showed abnormal growing patterns. In the grafting experiments in short days, scions of tuberizing genotypes on non-tuberizing rootstocks formed aberrant side-shoots. Although these structures were green and lacked the characteristic hook found on stolon tips, they resembled stolons (Fig. 8A-B). These "aerial stolons" grew towards the soil and in some cases, once reaching the soil, formed tubers at the tip (Fig. 8B). These stolon-like structures were also found in long days, in grafts with scions of the early-tuberizing CE3130 on non-tuberizing rootstocks. Stolon-like structures also formed on stems of potato plants in inducing short days (Fig. 8C-D), where stolons were removed. In some cases, tubers formed directly on the stem (Fig. 8D). Potato plants that were induced to tuberize, but unable to do so in the conventional way, found alternative means of tuberization.

\section{DISCUSSION}

\section{Grafting with non-tuberizing rootstocks did not improve flower bud development}

Long-day grafts with $S$. etuberosum rootstocks did not form tubers, but reached anthesis less often than the tuberizing control grafts and produced less open flowers when anthesis was 
reached (Fig. 3A-B). This is in line with results in opposite grafts, where the effect of tuberizing rootstocks on S. etuberosum scions was tested; in these grafts the fraction of plants with open flowers increased compared to control grafts with $S$. etuberosum rootstocks (Table 1). The results show that removing the tuber-sink does not improve flower bud development.

That tuberizing rootstocks did not impair, but improved flower development in S. etuberosum scions, was surprising. Instead of inhibiting flower development, tuberization may improve flowering in a different species (S. etuberosum). The FT of one species can induce flowering or tuberization in another species, for instance with rice Heading date $3 a$ ( $H d 3 a$ ) in potato, Arabidopsis FT in tomato and tobacco, and tomato SINGLE FLOWER TRUSS (SFT) in Arabidopsis (Lifschitz et al., 2006; Lifschitz and Eshed, 2006; Navarro et al., 2011). Potato StSP6A from the rootstock may improve S. etuberosum flowering in the scion. Interestingly, long-day grafts between S. etuberosum scions and leafless CE3027 or CE3130 rootstocks flowered and tuberized, while short-day grafts between $S$. etuberosum scions and the leafless CE3027 and CE3130 rootstocks did not (data not shown). Perhaps in long days, a leaf-derived FT from $S$. etuberosum induces tuberization, while in short days this signal is not produced. Potato plants are thought to have an auto regulatory StSP6A loop, where leaf-derived StSP6A leads to upregulation of StSP6A in the stolons, enhancing the level of StSP6A for tuberization (Navarro et al., 2011). Potato scions expressing rice Hd3a but no StSP6A have induced StSP6A in the stolons (Navarro et al., 2011). If FT from S. etuberosum also induces this auto regulatory loop, S. etuberosum FT may induce tuberization in the CE rootstocks and amplify the amount of FTs in the graft, possibly enhancing flowering as well.

Most importantly our grafting experiments show that the tuber-sink does not impair flower bud development. However, because interspecific grafts were used, effects on flowering may have been caused by other properties of the $S$. etuberosum than its inability to tuberize. Therefore, to determine whether removing the tuber-sink improves flower bud development, stolons were removed in potato plants.

\section{Removing stolons did not improve flower bud development}

As with grafting, removing the stolons did not improve flower bud development in both CE3027 and S. andigena genotypes (Table 2 and Fig. 6). This is in line with previous experiments on stolon abscission (Weinheimer and Woodbury, 1966). Removing stolons also had no effect on flower initiation. The lack of stolons did lead to an increase of assimilates available for the shoot, as seen in the significant increase in shoot biomass (Table 2). However, this increase in shoot biomass did not improve flower bud development. 
In short days, flower bud development was impaired compared to long days (Table 2). However, by raising short-day light intensity to match the light sum of long days, the fraction of plants to reach anthesis increased from two out of eleven plants $(<20 \%)$ to ten out of eleven plants (>90\%), which almost rivalled long-day anthesis (anthesis in all plants). Sufficient light is crucial for flower bud development, as has been demonstrated in several crops including potato and tomato (Kinet, 1977; Demagante and Zaag, 1988; Turner and Ewing, 1988). Increasing light may increase the amount of assimilates formed in the plant. Assimilates like sucrose play an important role in flower induction and floral development in potato (Chincinska et al., 2008). Nevertheless, the number of open flowers was significantly higher under longday conditions, indicating an impairment of CE3027 flowering in short days, as was found before in other potato genotypes (Turner and Ewing, 1988). Thus, short-day flower bud development was impaired and preventing formation of the tuber-sink by removal of the stolons did not improve this development.

Removing the tuber-sink in a plant that was induced to tuberize led to "aerial stolons"

Removing the tubers did not improve flower bud development. Although the plants were unable to tuberize, they were still induced to do so. Grafts that could not tuberize, started to produce stolon and tuber-like structures on the scions (Fig. 8A-B). Plants without stolons, growing in short days, also made stolon-like structures on the stem (Fig. 8C-D). Alternative tuberization structures have been documented before (Thijn, 1954; Weinheimer and Woodbury, 1966) in conditions where tuberization is prevented but plants remain induced to tuberize. The lack of tubers led to more assimilates in the shoot, but instead of promoting flowering these assimilates may have been directed towards alternative tuberization structures. The tuberization signal StSP6A is still expressed in inducing conditions, even when tubers are removed, which may be the cause of the direction of assimilates to alternative tuberization structures instead of to the flower buds. This theory is supported by the finding that the formation of stolon-like structures in short-day StSP6A RNAi plants was much less severe.

\section{The tuberization signal StSP6A impairs flower bud development}

$S$. andigena wild-type plants underwent floral transition in all tested light treatments, but in short days the flower buds ceased to develop at a very early stage (Fig. 5A). Remarkably, shortday flower bud development was significantly improved in the StSP6A RNAi lines. Two of the StSP6A RNAi plants were even able to reach anthesis in short days, which did not happen in any other treatment and is uncommon for $S$. andigena when grown in our climate chamber 
conditions. Flowering in StSP6A RNAi plants was also tested by Navarro et al. (2011), but only transition to flowering was considered and not flower bud development. The transition to flowering occurred at the same time as in the wild type, as was the case in our experiments (data not shown). The transgenic lines had a significantly reduced StSP6A expression (Supplementary Fig. S2A). Improved flower bud development in the transgenic lines could not be explained by an increase in transcription of the proposed flowering signal StSP3D in the leaves (data not shown), implying that StSP6A negatively affects flower bud development through a different mechanism.

StSP3D has been proposed to be the flowering signal in potato, because silencing StSP3D showed a late flowering response (Navarro et al., 2011). However, there is a lack of correlation between flower bud development and $S t S P 3 D$ expression, which is strongly expressed under short day conditions but weakly expressed under long day conditions (in prep. Dr. S. Bergonzi). Perhaps low expression levels of StSP3D are sufficient to induce flowering and the level of StSP6A determines the success of flower bud development. To fully understand potato flowering, elucidating the role of StSP6A in flower bud development, as well as StSP3D in flowering time and development, will be crucial.

Our finding that StSP6A represses flower bud development, while the tuber-sink does not, was confirmed in another experiment testing both stolon abscission (tuber-sink) and silencing of StSP6A (tuberization signal) in S. andigena in short days. Removal of stolons did not improve flower bud development, while downregulation of StSP6A did. The repressing role of StSP6A on flower bud development was further confirmed in CE3027 StCDF1 overexpressing lines, with upregulated StSP6A in long days. The flower bud development was impaired in these lines and resembled the impaired flower bud development found in wild-type $S$. andigena plants in short days. Transgenic lines in which an upstream regulator of StSP6A was overexpressed were used instead of StSP6A overexpressing lines, to induce StSP6A in long days. In Navarro (2011), transgenic lines overexpressing StSP6A actually improved flowering, perhaps by the strong and ubiquitous expression of StSP6A by the 35S promotor (Odell et al., 1985; Seternes et al., 2016). In the $S t C D F 1$ overexpressing lines, the down-stream regulation on StSP6A is still intact, allowing a more realistic upregulation of StSP6A than in a $35 S:: S t S P 6 A$ overexpressing line. Flower impairment in these lines confirms our earlier findings that StSP6A represses flower bud development.

Can the inhibiting effect of a tuber-sink be ruled out? 
542 Although flower bud development was not improved by tuber-sink removal in CE3027,

543 CE3130 or S. andigena, removing the tuber-sink had a positive effect on flowering in some genotypes in the past (Thijn, 1954; Jessup, 1958). However, these reports have also been

545

546

547

548

549

550

551

552

553

554

555

556

557

558

559

560

561

562

563

564

565

566

567

568

569

570

571

572

573

574

575 contradicted (Turner and Ewing, 1988). Therefore, it may be possible that repression of flower development by the tuber-sink is genotype specific. It would be interesting to find out if reducing StSP6A would further improve flower development in genotypes that are benefitted by tuber-sink removal. Nevertheless, our findings show that in S. andigena and CE3027 the tuber-sink does not repress flower bud development while the tuberization signal StSP6A does.

\section{The day-length control of flowering in potato}

Short days, or more correctly long nights, induce tuberization in potatoes, although variation exists between varieties in their dependence on short days (Garner and Allard, 1923; Ewing and Struik, 1992; Prat, 2010; Kloosterman et al., 2013). Potato flowering has been categorized as a short-day, long-day and day-neutral process (Jones and Borthwick, 1938; Turner and Ewing, 1988; Almekinders and Struik, 1994; Martínez-García et al., 2002; Schittenhelm et al., 2004). A cause for this variation might be the use of different genotypes and the difference in defining flowering. Because flowering is a process composed of many phases, it needs a clear distinction when addressed: it starts with flower transition and proceeds with flower bud and organ development. More importantly, the flowering process is not only influenced by day length but by tuberization as well, which varies between genotypes. Our results show that the floral transition occurs independently of the photoperiod but that flower bud development is repressed by the tuberization signal. Remarkably anthesis was only attained in short days with high irradiance (in StSP6A RNAi\#13) indicating that environmental growing conditions also play a role in the process. Our results point to a short-day control of flower bud development in potato, but due to internal control by StSP6A, flower bud development is promoted under long-day conditions.

\section{Interaction between two modes of reproduction in potato}

A likely mode of action for StSP6A to impair flower bud development, could be through control of assimilates. Although removing the tuber-sink did not improve flower bud development, it cannot be claimed assimilates do not play a role, as alternative tuber structures that acted as sinks were still formed unless StSP6A was silenced. StSP6A may have a role in directing assimilates towards tuberization, which consequentially could be detrimental for flower development, especially if tuberization takes place while flower buds are still 
576 developing. How this direction of assimilates takes place remains to be elucidated. Whether

577 flowering is actually repressed by StSP6A may be genotype specific and depend on the timing 578 of both tuberization and flowering. The European Cultivated Potato Database 579 (https://www.europotato.org) shows a huge variation in flowering success between varieties 580 and it has been suggested that potato berry and seed development is impeded by earliness of 581 tuberization (Pallais, 1987). Similar findings were seen in the CE3027 and CE3130 control 582 grafts, where the early tuberizing CE3130 grafts flowered less profusely than the later 583 tuberizing CE3027 grafts (Fig. 3B and Supplementary Table S2). It would be interesting to 584 correlate the tuberization time and StSP6A expression to the flowering time and flower 585 developmental success in a large number of genotypes.

586 While two reproduction modes may inhibit each other in the same species, interspecies 587 interaction between reproduction modes may be beneficial for both processes, as was seen in 588 S. etuberosum scions grafted on CE3027 and CE3130 rootstocks. The flowering in $S$. 589 etuberosum scions was improved compared to control grafts with S. etuberosum rootstocks. 590 StSP6A may not function as an inhibiter in S. etuberosum because flowering and tuberization 591 are not competing processes in this species. Consequently, StSP6A may substitute FT in $S$. 592 etuberosum and improve flowering, while StSP6A inhibits flowering in potato.

Conclusion

595 Our results show that flower bud development in potato is impaired by the tuberization signal StSP6A, and not by the tuber-sink itself. These results suggest there is an internal mechanism in potato plants where one mode of reproduction can affect the other.

\section{SUPPLEMENTARY DATA}

601

602

Table S1. Overview of the five experiments testing how tuberization affects potato flower bud 603 development.

604 Table S2. Flower bud development and tuberization in grafts between CE3130 and $S$. 605 etuberosum.

606

607 Figure S1. StNAC expression ( $\mathrm{Ct}=$ cycle threshold) in wild-type S. andigena, StSP6A RNAi 608 \#1, and StSP6A RNAi \#13.

609

Figure S2. StSP6A expression and tuberization time in wild-type S. andigena, StSP6A RNAi 


\section{ACKNOWLEDGEMENTS}

614

615 We thank the Centre of Genetic Resources (CGN) seedbank for providing Solanum etuberosum 616 and Solanum palustre seeds. Special thanks to Priscila Malcolm Matamoros for help with the 617 grafting and Simonetti and Beekenkamp Plants for providing grafting clips. This research is 618 part of the FlowerPot project which was supported by the Division for Earth and Life Sciences 619 (ALW) with financial aid from the Netherlands Organization for Scientific Research (NWO), 620 Signify, Solynta, Beekenkamp Plants and Incotec. We thank Signify for providing the LEDs. 


\section{REFERENCES}

Abelenda JA, Navarro C, Prat S. 2011. From the model to the crop: genes controlling tuber formation in potato. Current Opinion in Biotechnology, 22, 287-92.

Abelenda JA, Cruz-oro E, Franco-zorrilla M, Prat S. 2016. Potato StCONSTANS-like1

Suppresses Storage Organ Formation by Directly Activating the FT-like StSP5G Repressor. Current Biology, 26, 872-881.

Almekinders CJM, Struik PC. 1994. Photothermal response of sympodium development and flowering in potato (Solanum tuberosum L.) under controlled conditions. Netherlands Journal of Agricultural Science, 42, 311-329.

Almekinders CJM, Struik PC. 1996. Shoot development and flowering in potato (Solanum tuberosum L.). Potato Research, 39, 581-607.

Andrés F, Coupland G. 2012. The genetic basis of flowering responses to seasonal cues. Nature Reviews Genetics, 13, 627-639.

Batutis EJ, Ewing EE. 1982. Far-red reversal of red light effect during long-night induction of potato (Solanum tuberosum L.) tuberization. Plant Physiology, 69, 672-674.

Chincinska IA, Liesche J, Krügel U, Michalska J, Geigenberger P, Grimm B, Kühn C. 2008. Sucrose transporter StSUT4 from potato affects flowering, tuberization, and shade avoidance response. Plant Physiology, 146, 515-528.

Demagante AL, Zaag P. 1988. The response of potato (Solanum spp.) to photoperiod and light intensity under high temperatures. Potato Research, 31, 73-83.

Ewing EE, Struik PC. 1992. Tuber formation in potato: induction, initiation, and growth. Horticultural Reviews, 14, 89-198.

Fu J, Yang L, Dai S. (2014). Identification and characterization of the CONSTANS-like gene family in the short-day plant Chrysanthemum lavandulifolium. Molecular Genetics and Genomics, 290, 1039-1054.

Garner WW, Allard HA. 1923. Further studies in photoperiodism, the response of the plant to relative length of day and night. Journal of Agricultural Research, 23, 871-959.

González-Schain ND, Díaz-Mendoza M, Żurczak M, Suárez-López P. 2012. Potato CONSTANS is involved in photoperiodic tuberization in a graft-transmissible manner. The Plant Journal, 70, 678-690.

Jackson SD. 1999. Multiple signaling pathways control tuber induction in potato. Plant Physiology, 119, 1-8.

Jessup RJ. 1958. Potato breeding in New South Wales. The Journal of the Australian Institute 
of Agricultural Science, 6, 21-26.

Jones HA, Borthwick HA. 1938. Influence of photoperiod and other factors on the formation of flower primordia in the potato. The American Potato Journal, 15, 331-336.

Karimi M, Inze D, Depicker A. 2002. GATEWAY vectors for Agrobacterium-mediated plant transformation. Trends in Plant Science, 7, 193-195.

Kinet JM. 1977. Effect of light conditions on the development of the inflorescence in tomato. Scientia Horticulturae, 6, 15-26.

Kloosterman B, Abelenda JA, del Mar Carretero Gomez M, Oortwijn M, de Boer JM, Kowitwanich K, Horvath BM, van Eck HJ, Smaczniak C, Prat S, Visser RGF, Bachem CWB. 2013. Naturally occurring allele diversity allows potato cultivation in northern latitudes. Nature. 495, 246-250

Krantz FA. 1939. Incidence and inheritance of pollen sterility in the potato. Journal of Agricultural Research, 58, 593-602.

Krauss A, Marschner H. 1984. Growth rate and carbohydrate metabolism of potato tubers exposed to high temperatures. Potato Research, 27, 297-303.

Lifschitz E, Eshed Y. 2006. Universal florigenic signals triggered by FT homologues regulate growth and flowering cycles in perennial day-neutral tomato. Journal of Experimental Botany, 57, 3405-3414.

Lifschitz E, Eviatar T, Rozman A, Shalit A, Goldshmidt A, Amsellem Z, Alvarez JP, Eshed Y. 2006. The tomato FT ortholog triggers systemic signals that regulate growth and flowering and substitute for diverse environmental stimuli. Proceedings of the National Academy of Sciences of the United States of America, 103, 6398-6403.

Lindhout P, Meijer D, Schotte T, Hutten RCB, Visser RGF, van Eck HJ. 2011. Towards F1 Hybrid Seed Potato Breeding. Potato Research, 54, 301-312.

Martínez-García JF, Virgós-Soler A, Prat S. 2002. Control of photoperiod-regulated tuberization in potato by the Arabidopsis flowering-time gene CONSTANS. Proceedings of the National Academy of Sciences of the United States of America, 99, 15211-15216.

Murashige T, Skoog F. 1962. A Revised Medium for Rapid Growth and Bio Assays with Tobaoco Tissue Cultures. Physiologia Plantarum, 15, 473-497.

Navarro C, Abelenda JA, Cruz-Oró E, Cuéllar CA, Tamaki S, Silva J, Shimamoto K, Prat S. 2011. Control of flowering and storage organ formation in potato by FLOWERING LOCUS T. Nature, 478, 119-123.

Navarro C, Cruz-Oró E, Prat S. 2015. Conserved function of FLOWERING LOCUS T (FT) homologues as signals for storage organ differentiation. Current Opinion in Plant Biology, 23, 
$45-53$.

Pallais N. 1987. True potato seed quality. Theoretical and Applied Genetics, 73, 784-792.

Plantenga FDM, Siakou M, Bergonzi S, Heuvelink E, Bachem CWB, Visser RGF, Marcelis LFM. 2016. Regulating flower and tuber formation in potato with light spectrum and day length. Acta Horticulturae, 1134, 267-276.

Potato Genome Sequencing Consortium. 2011. Genome sequence and analysis of the tuber crop potato. Nature, 475, 189-195.

Prat S. 2010. Hormonal and daylength control of potato tuberization. In: Davies PJ, ed. Plant Hormones. Dordrecht: Springer, 574-596.

Rodríguez-Falcón M, Bou J, Prat S. 2006. Seasonal control of tuberization in potato: conserved elements with the flowering response. Annual Review of Plant Biology, 57, 151180.

Schittenhelm S, Menge-Hartmann U, Oldenburg E. 2004. Photosynthesis, Carbohydrate Metabolism, and Yield of Phytochrome-B-Overexpressing Potatoes under Different Light Regimes. Crop Science, 44, 131-143.

Sweetlove LJ, Kossmann J, Riesmeier JW, Trethewey RN, Hill SA. 1998. The control of source to sink carbon flux during tuber development in potato. The Plant Journal, 15, 697-706. Thijn GA. 1954. Observations on flower induction with potatoes. Euphytica, 3, 28-34.

Tsuji H, Taoka KI, Shimamoto K. 2011. Regulation of flowering in rice: Two florigen genes, a complex gene network, and natural variation. Current Opinion in Plant Biology, 14, 45-52.

Turner AD, Ewing EE. 1988. Effects of photoperiod, night temperature, and irradiance on flower production in the potato. Potato Research, 31, 257-268.

Visser RGF. 1991. Regeneration and transformation of potato by Agrobacterium tumefaciens. In: Lindsey K, ed. Plant Tissue Culture Manual. Dordrecht: Springer, 301-309.

Weinheimer WH, Woodbury GW. 1966. Effects of grafting and solanum understocks on flower formation and abscission of flowers and fruits in the Russet Burbank potato. American Potato Journal, 43, 453-457.

Zaheer K, Akhtar MH. 2016. Potato Production, Usage, and Nutrition - A Review. Critical Reviews in Food Science and Nutrition, 56, 711-721. 
Table 1. Flower bud development and tuberization in grafts with $S$. etuberosum scions and S. etuberosum or CE3027 rootstocks (with or without leaves) (Exp. 1).

\begin{tabular}{|c|c|c|c|c|c|}
\hline & Rootstock & $n$ & $\begin{array}{c}\text { Anthesis } \\
*\end{array}$ & $\begin{array}{c}\text { Max. open } \\
\text { flowers/plant }\end{array}$ & $\begin{array}{c}\text { Tuber dry weight } \\
\text { (g/plant) }\end{array}$ \\
\hline \multirow{3}{*}{ 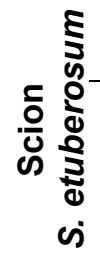 } & S. etuberosum (non-tuberizing) & 3 & 1 & $3.3 \pm 3.1 a^{* *}$ & $0.00 \pm 0.00 \mathrm{a}$ \\
\hline & CE3027 (tuberizing) & 9 & 3 & $3.0 \pm 3.3 \mathrm{a}$ & $1.03 \pm 1.95 \mathrm{a}$ \\
\hline & CE3027 + leaves (tuberizing) & 10 & 5 & $1.7 \pm 3.1 \mathrm{a}$ & $1.77 \pm 1.96 \mathrm{a}$ \\
\hline
\end{tabular}

* Plants that reached the open flower stage

** Standard deviations are given, identical alphabetical letters indicate no significant difference between graft combinations $(\alpha=0.05)$ 
Table 2. The effect of removing stolons on CE3027 flowering and plant biomass in different light treatments. Biological replicates, $n=11$. (Exp. 2).

\begin{tabular}{llccccc}
\hline $\begin{array}{l}\text { Light } \\
\text { treatment }\end{array}$ & Stolons & $\begin{array}{c}\text { Anthesis } \\
*\end{array}$ & $\begin{array}{c}\text { Max. open } \\
\text { flowers/plant }\end{array}$ & $\begin{array}{c}\text { Tuber dry } \\
\text { weight } \\
\text { (g/plant) }\end{array}$ & $\begin{array}{c}\text { Shoot dry } \\
\text { weight } \\
\text { (g/plant) }\end{array}$ & $\begin{array}{c}\text { Shoot + tuber } \\
\text { dry weight } \\
\text { (g/plant) }\end{array}$ \\
\hline \multirow{2}{*}{ SD200** } & Intact & 2 & $0.3 \pm 0.6 \mathrm{a}$ a** & $4.2 \pm 0.9 \mathrm{~b}$ & $2.2 \pm 0.2 \mathrm{a}$ & $6.5 \pm 1.0 \mathrm{~b}$ \\
& Removed & 2 & $0.2 \pm 0.4 \mathrm{a}$ & $0.0 \pm 0.0 \mathrm{a}$ & $5.5 \pm 0.9 \mathrm{c}$ & $5.5 \pm 0.9 \mathrm{a}$ \\
\hline \multirow{2}{*}{ SD400 } & Intact & 10 & $1.2 \pm 1.0 \mathrm{ab}$ & $10.1 \pm 1.1 \mathrm{C}$ & $2.4 \pm 0.2 \mathrm{ab}$ & $12.5 \pm 1.2 \mathrm{c}$ \\
& Removed & 10 & $2.4 \pm 1.0 \mathrm{~b}$ & $0.0 \pm 0.0 \mathrm{a}$ & $7.8 \pm 1.8 \mathrm{~d}$ & $7.8 \pm 1.8 \mathrm{~b}$ \\
\hline \multirow{2}{*}{ LD200 } & Intact & 11 & $6.5 \pm 1.5 \mathrm{C}$ & $4.1 \pm 1.4 \mathrm{~b}$ & $3.5 \pm 0.3 \mathrm{~b}$ & $7.6 \pm 1.5 \mathrm{~b}$ \\
& Removed & 11 & $6.2 \pm 1.0 \mathrm{C}$ & $0.0 \pm 0.0 \mathrm{a}$ & $7.8 \pm 1.1 \mathrm{~d}$ & $7.8 \pm 1.1 \mathrm{~b}$ \\
\hline
\end{tabular}

* The number of plants that reached the open flower stage

** Number indicates light intensity in $\mu \mathrm{mol} \cdot \mathrm{m}^{-2} \cdot \mathrm{s}^{-1}, \mathrm{SD}=$ short day ( 8 hours), LD = long day (16 hours)

*** Standard deviations are given, identical alphabetical letters indicate no significant difference between treatments $(\alpha=0.05)$ 
Figure 1. The three methods used to eliminate tuberization in potato and determine whether flower bud development is improved. (1) Potato plant scions that are able to tuberize are grafted onto non-tuberizing wild potato rootstocks (2) The stolons of the potato plant are removed as soon as they appear. (3) The tuberization signal StSP6A, which is expressed in the leaves, is silenced in transgenic lines.

Figure 2. Schematic representation of flowering and tuberization in the grafting combinations between a tuberizing and non-tuberizing genotype, in long days. Potato genotype CE3027 is able to tuberize, while S. etuberosum is unable to tuberize. (A) The control grafts of CE3027 made tubers. (B) Grafts with CE3027 scions and S. etuberosum rootstocks did not make tubers. (C) The control graft of S. etuberosum did not make tubers. (D) Grafts with S. etuberosum scions and CE3027 rootstocks did make tubers, with or without leaves on the rootstock. All graft combinations formed buds which developed into open flowers. The graft combinations with a tuberizing rootstock (A, D) formed more open flowers than grafts without tuberizing rootstocks (B, C). (Exp. 1).

Figure 3. Flower bud development and tuber biomass in grafts with potato scions (CE3027) and tuberizing rootstocks (CE3027) or non-tuberizing rootstocks (S. etuberosum) in long days. (A) The percentage of grafts that reached anthesis (open flowering stage), absolute numbers are indicated in the bar. (B) The maximum number of open flowers on a plant. (C) The dry weight of the tubers per plant at harvest, NT = no tuberization (biomass $0)$. The asterisk represents a significant difference between grafts with a tuberizing rootstock and a non-tuberizing rootstock, $\alpha=0.05$. Error bars show standard deviations. (Exp. 1). $n=11$ (CE3027/CE3027) and $n=8($ CE3027/S. etuberosum).

Figure 4. Flower buds in $\boldsymbol{S}$. andigena in short and long days. Flower buds in high-light short days (SD400, 8/16 hours light/dark, $400 \mu \mathrm{mol} \cdot \mathrm{m}^{-2} \cdot \mathrm{s}^{-1}$ ) and low-light long days (LD200, 16/8 hours light/dark, $200 \mu \mathrm{mol} \cdot \mathrm{m}^{-2} \cdot \mathrm{s}^{-1}$ ) five weeks after transplanting and eight weeks after transplanting. (Exp. 3).

Figure 5. The effect of photoperiod and light intensity on flower bud development in $S$. andigena wild-type and StSP6A RNAi plants. Four light treatments were used: SD200 (short day, 8/16 hours light/dark, $200 \mu \mathrm{mol} \cdot \mathrm{m}^{-2} \cdot \mathrm{s}^{-1}$ ), SD400 (short day, 8/16 hours light/dark, 400 $\mu \mathrm{mol} \cdot \mathrm{m}^{-2} \cdot \mathrm{s}^{-1}$ ), LD200 (long day, 16/8 hours light/dark, $200 \mu \mathrm{mol} \cdot \mathrm{m}^{-2} \cdot \mathrm{s}^{-1}$ ) and LD400 (long day, $16 / 8$ hours light/dark, $400 \mu \mathrm{mol} \cdot \mathrm{m}^{-2} \cdot \mathrm{s}^{-1}$ ). SD400 and LD200 have the same daily light sum. (A) Flower bud development was categorized by size where 0 was no bud and 5 was an open flower. The median of the furthest stage of bud development during growing is given. Identical letters 
indicate no significant difference between genotypes in a light treatment $(\alpha=0.05)$. Biological replicates $S$. andigena, $n=8$ and StSP6A RNAi lines, $n=5$. (B) Plants at harvest. A wild-type S. andigena in SD400, a StSP6A RNAi \#13 plant in SD400, a wild-type S. andigena in LD200 and a StSP6A RNAi \#13 plant in LD200. (Exp. 3).

Figure 6. The flower bud development in S. andigena in wild-type and StSP6A RNAi\#13 lines where the stolons were either left intact or removed. Plants were grown in short days (8/16 hours light/dark) with a light intensity of $400 \mu \mathrm{mol} \cdot \mathrm{m}^{-2} \cdot \mathrm{s}^{-1}$. (A) Flower bud size during growth (measurements were ceased when flower bud abortion started) and (B) maximum flower bud size reached by the plant. Error bars show standard deviations. Letters indicate significant differences in maximum flower bud size between treatments $(\alpha=0.05)$. Biological replicates, $n=10$. WT = wild type. (C) Flower buds six weeks after transplanting, in WT $S$. andigena and StSP6A RNAi\#13 S. andigena, with stolons intact or removed. (Exp. 4).

Figure 7. StSP6A expression and flowering phenotypes in wild-type CE3027, 35S::StCDF1 \#3 and 35S::StCDF1 \#4. Plants were grown in long days (16/8 hours light/dark) of $200 \mu \mathrm{mol} \cdot \mathrm{m}^{-2} \cdot \mathrm{s}^{-1}$ (A) StSP6A is expressed relative to the reference gene StNAC. Expression is from plants five weeks after transplanting. The error bars show the standard deviations. Alphabetical letters indicate significant differences between genotypes $(\alpha=0.05)$. Biological replicates, $n=4$. (B) The shoot apex in wild-type and transgenic CE3027 plants eight weeks after transplanting. Genotypes from left to right: wild type, 35S::StCDF1 \#3 and 35S::StCDF1 \#4. The number of plants that reached anthesis after eight weeks is indicated. (Exp. 5).

Figure 8. Stolon-like side-shoots formed under tuber inducing conditions if tuberization was impaired. (A) A graft where the scion of a plant that was able to tuberize was grafted onto a wild non-tuberizing rootstock (CE3027 / S. palustre) in short days. Stolon-like structures are formed above the graft unison (white arrow). (B) In the same graft combination the stolon-like structures on the scion formed a tuber upon reaching the soil. (C) In the CE3027 plants where the stolons were removed, stolon-like structures were formed aboveground on the stem. (D) $S$. andigena plants grown in short days where the stolons were removed, formed tuber-like structures directly on the stem. 

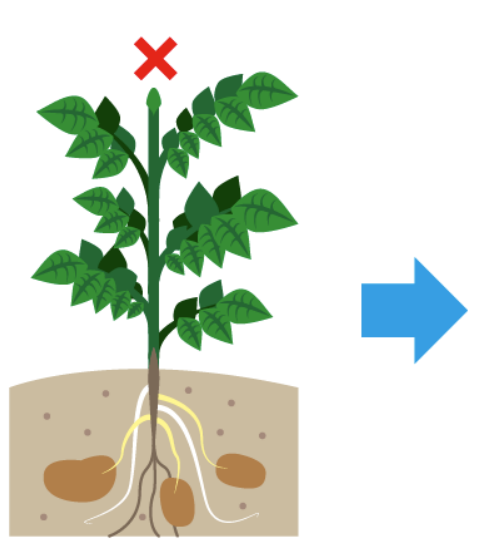

1

2

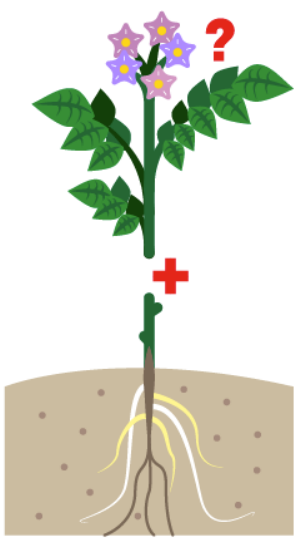

3
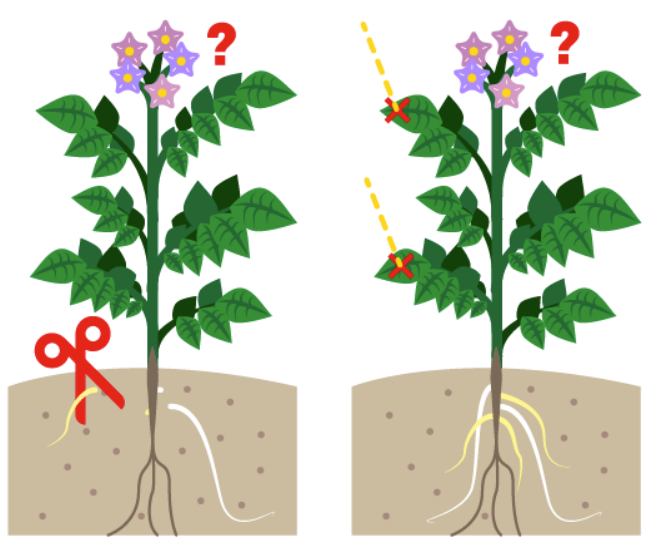

Figure 1. 
A

B

Tuberizing type

$\stackrel{+}{+}$ Tuberizing type

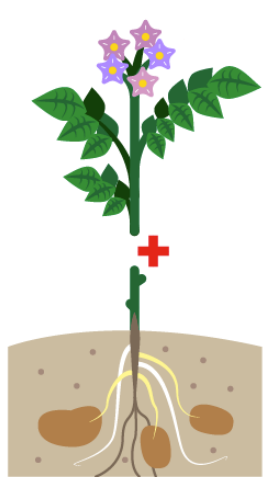

CE3027

CE3027
Tuberizing type

$+$

Non-tuberizing type

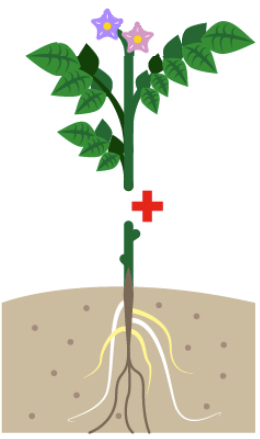

CE3027

S. etuberosum
C

Non-tuberizing type $+$

Non-tuberizing type

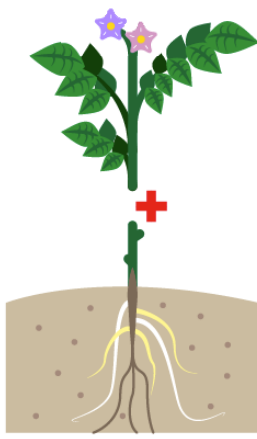

S. etuberosum
D

Non-tuberizing type

Tuberizing type

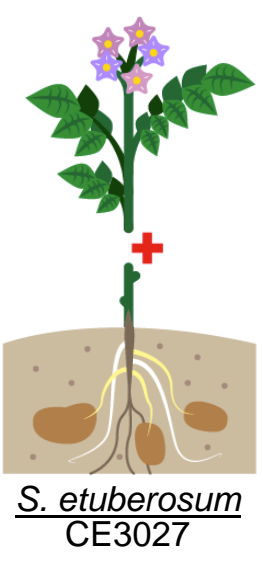

Figure 2. 
A

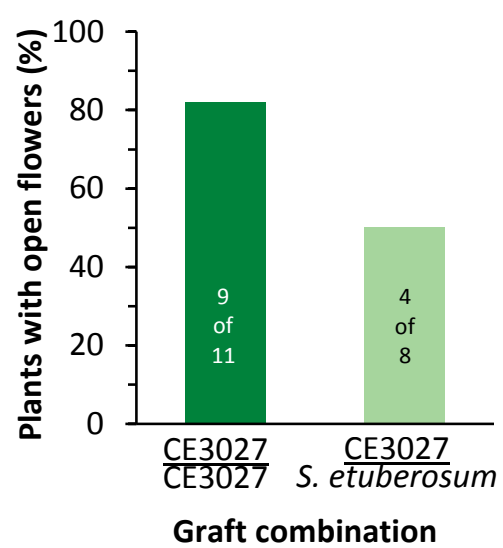

B

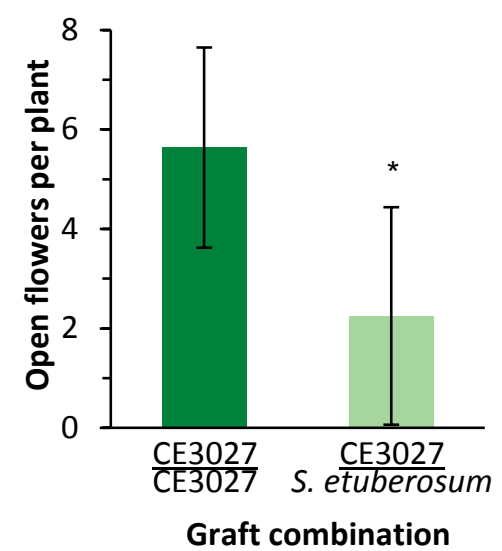

C

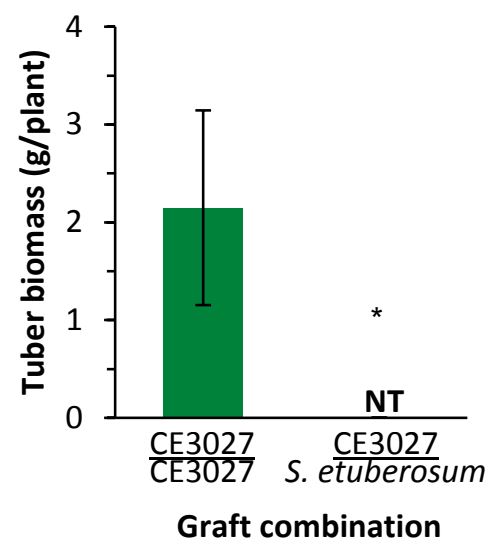

Figure 3. 

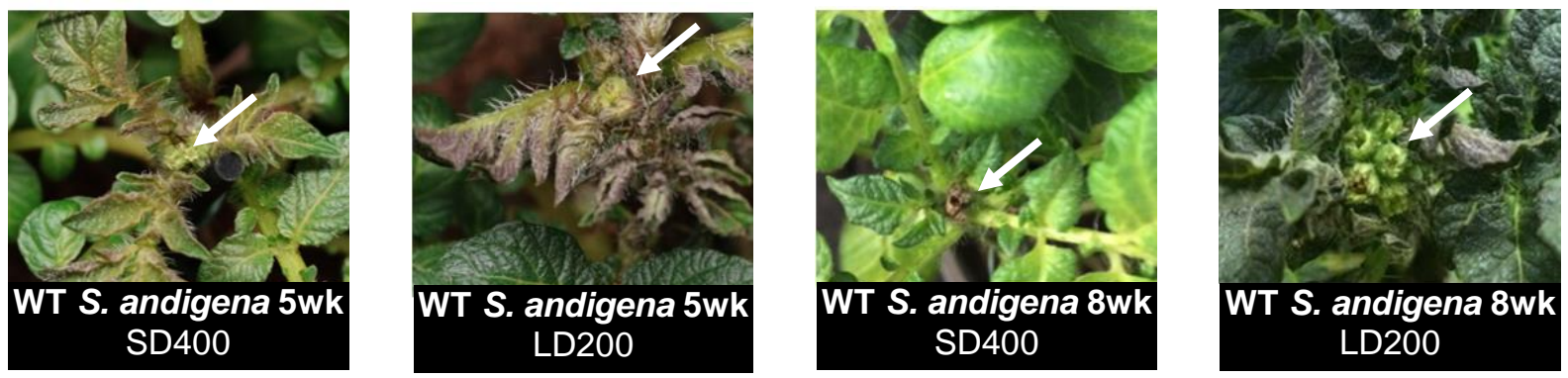

Figure 4. 
A

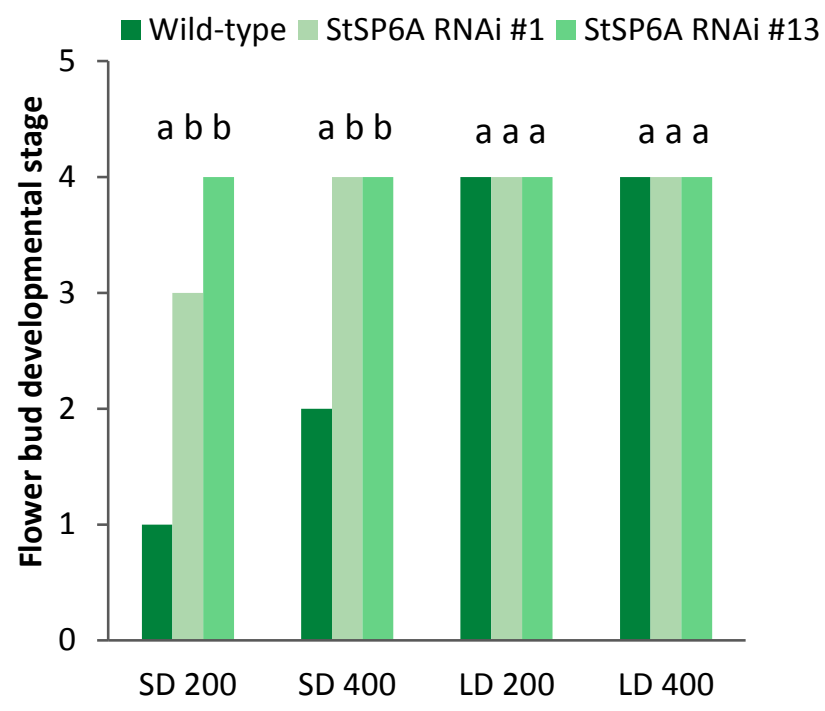

B
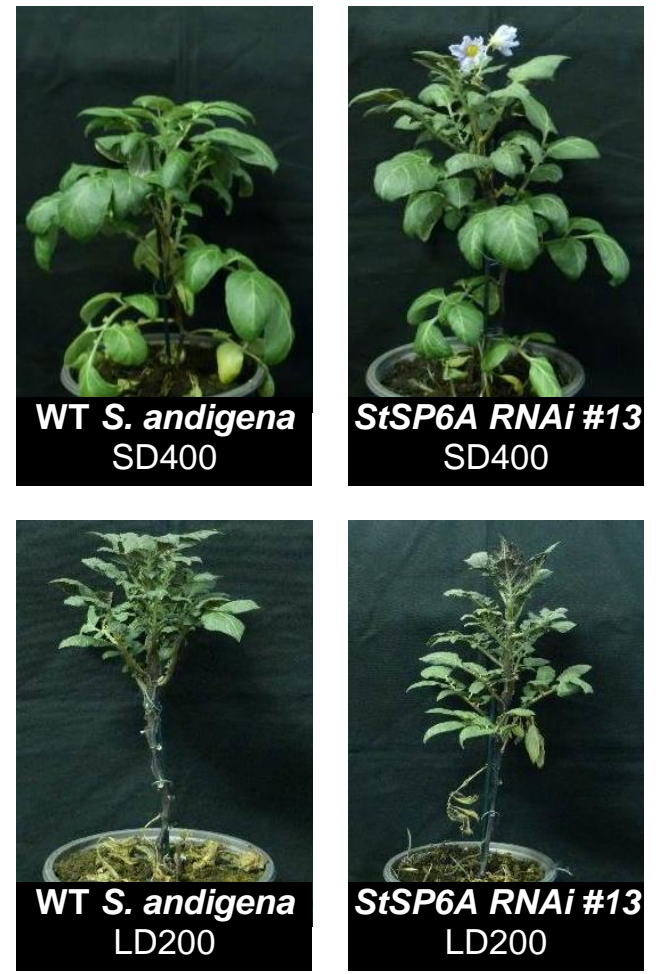

Figure 5. 
C

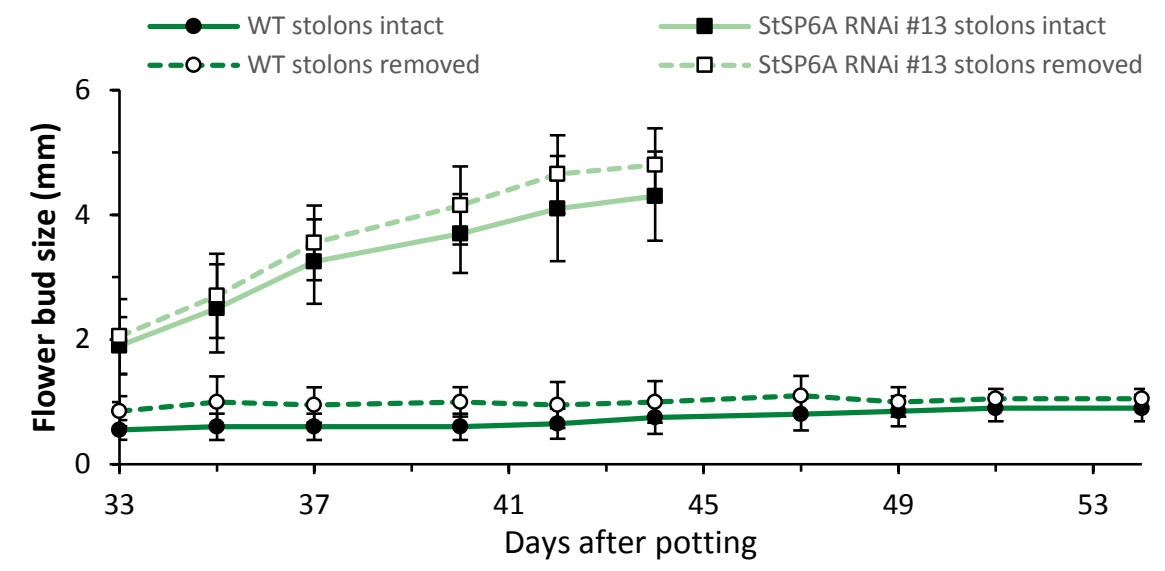

B

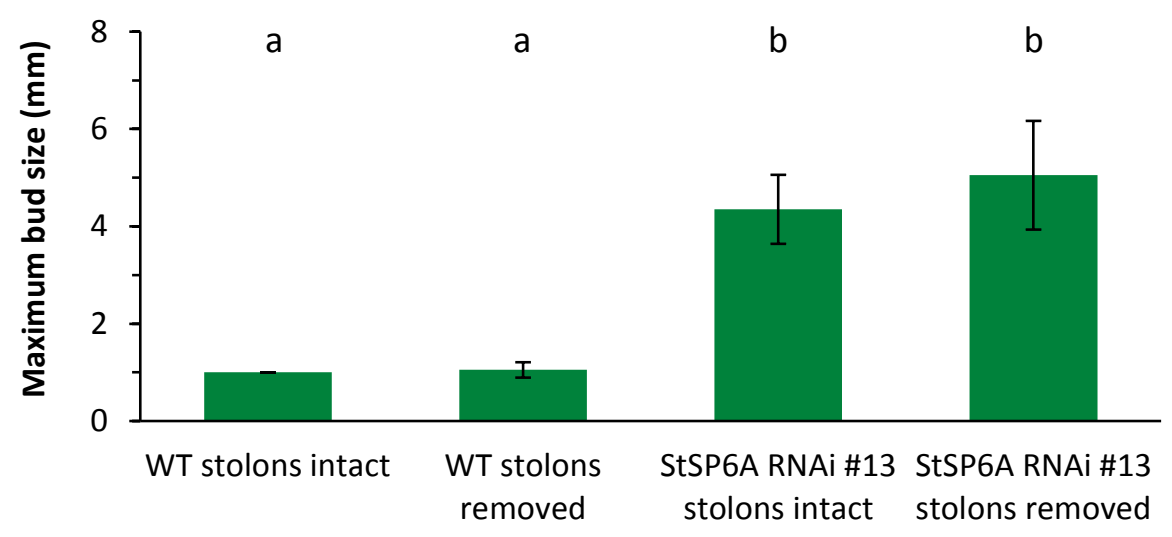

StSP6A RNAi

no stolons

\section{Figure 6.}


A

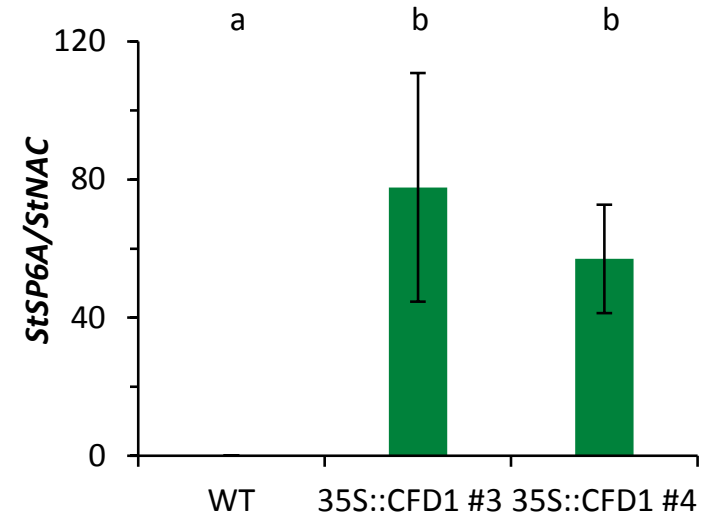

B

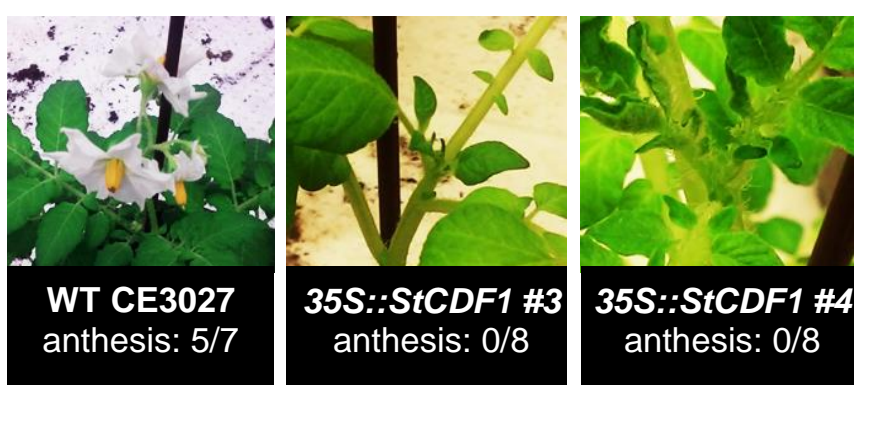

Figure 7. 

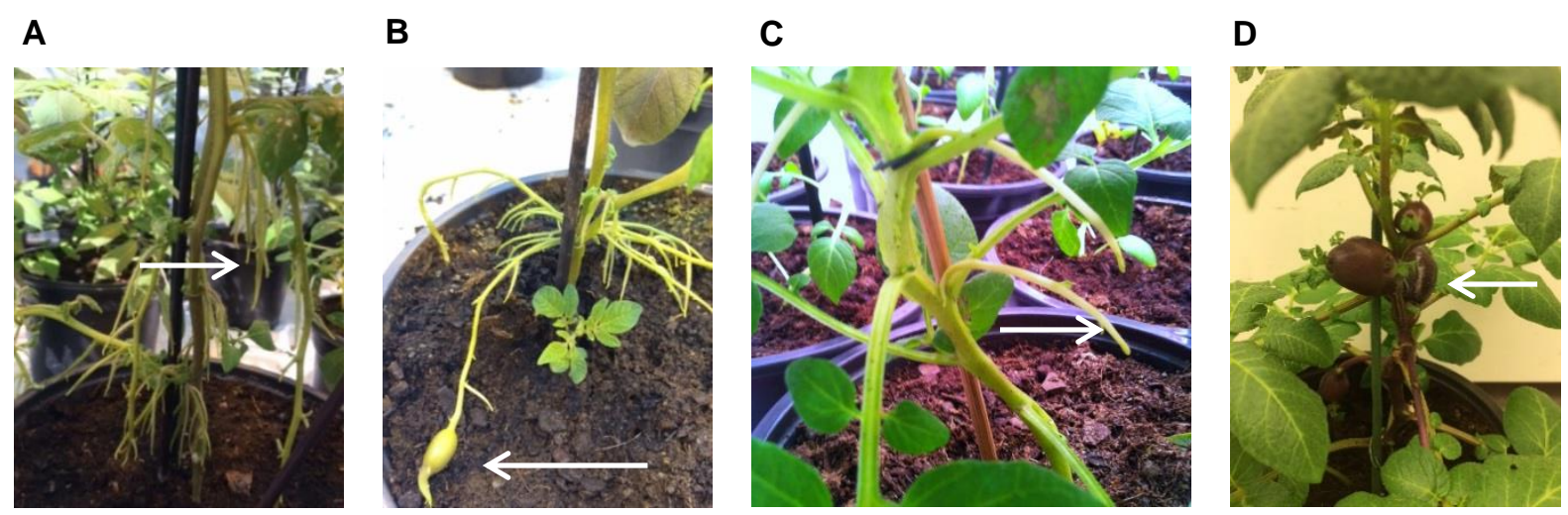

Figure 8. 


\section{SUPPLEMENTARY DATA}

Table S1. Overview of the five experiments testing how tuberization affects potato flower bud development.

\begin{tabular}{|c|c|c|c|c|}
\hline Exp. & $\begin{array}{l}\text { Method of tuber } \\
\text { removal }\end{array}$ & $\begin{array}{l}\text { Plants per } \\
\text { treatment }\end{array}$ & Genotypes & $\begin{array}{l}\text { Conditions: } \\
\text { day-length (hours), day/night } \\
\text { temperature }\left({ }^{\circ} \mathrm{C}\right) \text {, light intensity } \\
\left(\mu \mathrm{mol} \cdot \mathrm{s}^{-1} \cdot \mathrm{m}^{-2}\right) \text {, relative humidity, } \\
\text { duration (weeks), location }\end{array}$ \\
\hline $1 \mathrm{a}$ & $\begin{array}{l}\text { Removing } \\
\text { tuber-sink: } \\
\text { Grafting }\end{array}$ & 10 & $\begin{array}{l}\text { CE3027 } \\
\text { S. etuberosum } \\
\text { S. palustre }\end{array}$ & $\begin{array}{l}8,22 / 18^{* *}, \text { not measured (winter- } \\
\text { spring } 2016), \text { not measured, } 14, \\
\text { greenhouse }\end{array}$ \\
\hline $1 \mathrm{~b}$ & $\begin{array}{l}\text { Removing } \\
\text { tuber-sink: } \\
\text { Grafting }\end{array}$ & $10^{*}$ & $\begin{array}{l}\text { CE3027 } \\
\text { CE3130 } \\
\text { S. etuberosum }\end{array}$ & $\begin{array}{l}16-17,22 / 18^{\star *}, \text { not measured } \\
\text { (summer } 2016), \text { not measured, } 12, \\
\text { greenhouse }\end{array}$ \\
\hline 2 & $\begin{array}{l}\text { Removing } \\
\text { tuber-sink: Removing } \\
\text { stolons }\end{array}$ & 11 & CE3027 & $\begin{array}{l}8 \& 16,20 / 20,200 \text { and } 400,70 \%, 8, \\
\text { climate chamber }\end{array}$ \\
\hline 3 & $\begin{array}{l}\text { Removing tuber } \\
\text { signal: } \\
\text { Silencing StSP6A }\end{array}$ & $\begin{array}{l}8 \\
5 \\
5\end{array}$ & $\begin{array}{l}\text { S. andigena WT } \\
\text { StSP6A RNAi\#1 } \\
\text { StSP6A RNAi\#13 }\end{array}$ & $\begin{array}{l}8 \& 16,22 / 18,200 \text { and } 400,70 \%, 8, \\
\text { climate chamber }\end{array}$ \\
\hline 4 & $\begin{array}{l}\text { Removing tuber } \\
\text { signal and sink: } \\
\text { Silencing StSP } 6 A \text { and } \\
\text { removing stolons }\end{array}$ & 10 & $\begin{array}{l}\text { S. andigena } \\
\text { StSP6A RNAi\#13 }\end{array}$ & $\begin{array}{l}8,22 / 18,400,70 \%, 8, \text { climate } \\
\text { chamber }\end{array}$ \\
\hline 5 & $\begin{array}{l}\text { Increasing the tuber } \\
\text { signal: } \\
\text { Overexpressing } \\
\text { StCDF1 }\end{array}$ & $\begin{array}{l}10 \\
8 \\
8\end{array}$ & $\begin{array}{l}\text { CE3027 } \\
\text { 35S::StCDF1 \#3 } \\
\text { 35S::StCDF1 \#4 }\end{array}$ & $\begin{array}{l}16,20 / 18,200,70 \%, 6 \text {, climate } \\
\text { chamber }\end{array}$ \\
\hline esults & grafting combinati & s exceede & or failed to reac & this number ( $n$ indicated in th \\
\hline
\end{tabular}


Table S2. Flower bud development and tuberization in grafts between CE3130 and $S$. etuberosum.

\begin{tabular}{|c|c|c|c|c|c|}
\hline $\bar{A}$ & Rootstock & $n$ & $\underset{*}{\text { Anthesis }}$ & $\begin{array}{c}\text { Maximum open } \\
\text { flowers/plant }\end{array}$ & $\begin{array}{c}\text { Tuber dry weight } \\
\text { (g/plant) }\end{array}$ \\
\hline \multirow{2}{*}{ 응 } & CE3130 (tuberizing) & 10 & 9 & $2.6 \pm 1.4 b^{\star *}$ & $8.0 \pm 1.1 \mathrm{~b}$ \\
\hline & S. etuberosum (non-tuberizing) & 9 & 1 & $0.4 \pm 1.3 \mathrm{a}$ & $0.0 \pm 0.0 \mathrm{a}$ \\
\hline
\end{tabular}

\begin{tabular}{|c|c|c|c|c|c|}
\hline B & Rootstock & $n$ & Anthesis & $\begin{array}{c}\text { Maximum open } \\
\text { flowers/plant }\end{array}$ & $\begin{array}{c}\text { Tuber dry weight } \\
\text { (g/plant) }\end{array}$ \\
\hline$\stackrel{5}{5}$ & S. etuberosum (non-tuberizing) & 3 & 1 & $3.3 \pm 3.1 \mathrm{a}$ & $0.0 \pm 0.0 \mathrm{a}$ \\
\hline 응 & CE3130 (tuberizing) & 11 & 4 & $3.0 \pm 3.0 \mathrm{a}$ & $2.3 \pm 1.5 \mathrm{a}$ \\
\hline$\dot{~}$ & CE3130 + leaves (tuberizing) & 12 & 5 & $3.8 \pm 2.6 \mathrm{a}$ & $6.5 \pm 2.3 b$ \\
\hline
\end{tabular}

* Plants that reached the open flower stage

** Standard deviations are given, identical alphabetical letters indicate no significant difference between graft combinations $(\alpha=0.05)$ 


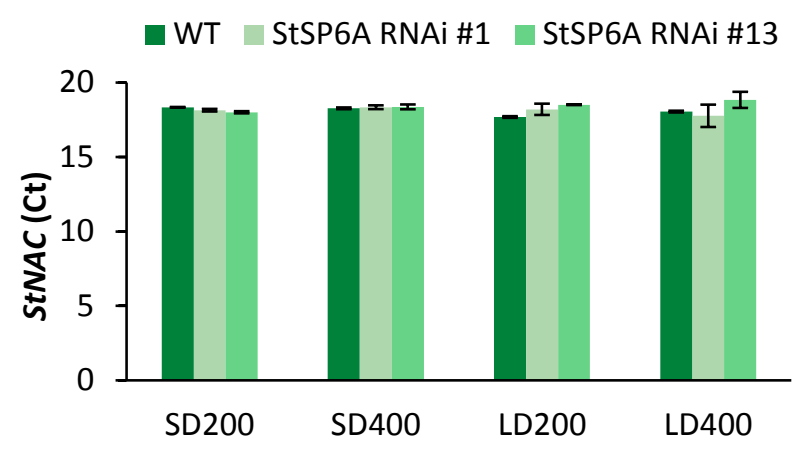

Figure S1. StNAC expression $(\mathrm{Ct}=$ cycle threshold $)$ in wild-type $S$. andigena, StSP6A $R N A i$ \#1, and StSP6A RNA $\boldsymbol{R}$ \#13. The reference gene StNAC is similarly expressed in all tested light treatments and genotypes. Plants were grown in short days (SD) of 200 and $400 \mu \mathrm{mol} \cdot \mathrm{m}^{-}$ ${ }^{2} \cdot \mathrm{s}^{-1}$ and long days (LD) of 200 and $400 \mu \mathrm{mol} \cdot \mathrm{m}^{-2} \cdot \mathrm{s}^{-1}$. The error bars show the standard deviation within a treatment/genotype. Technical repetitions, $n=3$. 

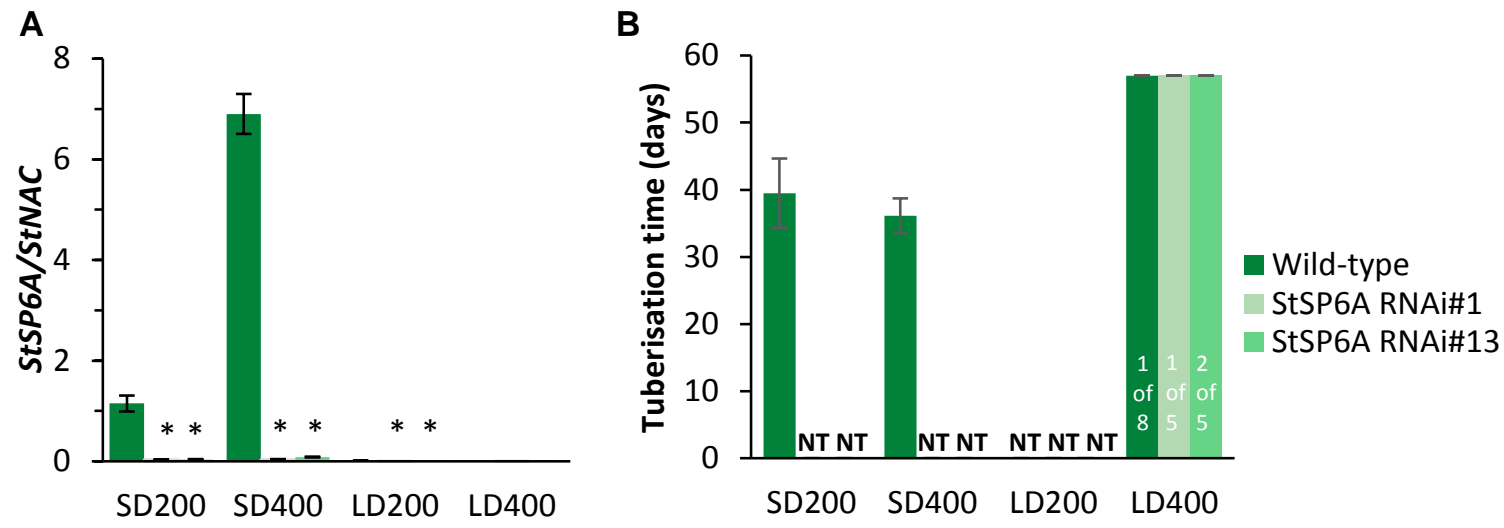

Figure S2. StSP6A expression and tuberization time in wild-type S. andigena, StSP6A $\boldsymbol{R N A i} \# 1$, and StSP6A RNAi \#13. Plants were grown in short days (SD) of 200 and 400 $\mu \mathrm{mol} \cdot \mathrm{m}^{-2} \cdot \mathrm{s}^{-1}$ and long days (LD) of 200 and $400 \mu \mathrm{mol} \cdot \mathrm{m}^{-2} \cdot \mathrm{s}^{-1}$. (A) StSP6A is expressed relative to the reference gene StNAC. Expression is from plants five weeks after transplanting. The error bars show the standard deviation. Asterisks indicate a significant difference to the wild-type expression in a given light treatment $(\alpha=0.05)$. Technical repetitions, $n=3$. (B) Tuberization time in days from transplanting to soil. The error bars show the standard deviation. The fraction of tuberizing plants is indicated in the bar (no indication means all plants tuberized). Biological replicates: Wild-type $(n=8)$, StSP6A RNAi \#1 $(n=5), \operatorname{StSP6A~RNAi\# 13~(n=5).~(Exp.~3).~}$ 